\title{
Complex ultrasound evaluation of non-alcoholic fatty liver disease. "One stop shop" with new ultrasound-based techniques.
}

\section{Zeno Sparchez}

$3^{\text {rd }}$ Medical Department, "Iuliu Hatieganu" University of Medicine and Pharmacy, Regional Institute for Gastroenterology and Hepatology, Cluj-Napoca, Romania

Nonalcoholic fatty liver disease (NAFLD) is currently a major health problem, affecting more than onequarter of adults in the world, $60 \%$ of diabetic patients and $90 \%$ of obese people having this condition [1]. The spectrum of NAFLD covers a wide histological variety from non-evolutive simple steatosis to progressive nonalcoholic steatohepatitis (NASH), which may progress to liver cirrhosis, hepatic failure and hepatocellular carcinoma (HCC) [1-3]. Patients with NAFLD experience a high risk of death from cardiovascular disease but also from end stage liver disease and HCC [1-4]. The risk increases significantly among patients with NASH and fibrosis [4].

Thus, key issues in NAFLD patients are the differentiation of NASH from simple steatosis and identification of advanced hepatic fibrosis. Liver biopsy has been the gold standard to differentiate simple steatosis from steatohepatitis with or without fibrosis [1-4]. However, it is an invasive diagnostic tool associated with rare but potentially life-threatening complications, has a poor acceptability which is relevant in such a big population, has sampling variability and is not suitable for follow up [3].

It has become obvious that in clinical practice for identifying NAFLD, it is preferable to use effective noninvasive methods for tracking disease processes and monitoring treatment effects [1-3]. Ultrasound (US) was the first-line imaging test used in clinical practice in individuals with suspected NAFLD [1]. Although it has a good accuracy for moderate-to-severe steatosis (sensitivity of $85 \%$ and specificity of $94 \%$ ) its value in detect-

Received Accepted

Med Ultrason

2021, Vol. 23, No 1, 5-6

Corresponding author: Zeno Sparchez MD, PhD

"O.Fodor" Institute for Gastroenterology and Hepatology,

Croitorilor 19-21, Cluj Napoca, Romania

Email: zsparchez@yahoo.co.uk ing steatosis of less than $20 \%$ is low $[1,5,6]$. Moreover, it cannot diagnose fibrosis and steatohepatitis, very important pathologic stages toward a severe liver disease. Another limitation of US is the impossibility to monitor disease progression and to assess various therapeutical methods $[1,2,5,6]$. Recent studies obtained better results using quantitative ultrasound (sonographic hepatorenal index, hepatic attenuation rate, Steatoscore, acoustic structure quantification [3-5]). Nevertheless, European guidelines for the management of NAFLD recommend the use of US as the first-choice imaging tool in adults at risk for NAFLD [3].

Elastography based techniques - Transient Elastography (TE) FibroScan, point Shear Wave Elastography (pSWE) and Two-Dimensional Shear Wave Elastography (2D-SWE) [5-9] - are used for the assessment of liver fibrosis in many liver diseases including NAFLD [1-5]. Using a XL probe FibroScan yields an AUROC of 0.82 for significant fibrosis, 0.86 for advanced fibrosis, and 0.94 for cirrhosis in a group of patients with NAFLD [1]. 2D-SWE was also able to detect advanced fibrosis and cirrhosis with high accuracy (88-92\% and $89-93 \%$ respectively) [3]. The accuracy of 2D SWE for diagnosing advanced fibrosis was found to be significantly higher than TE (AUROC difference of 12\%; $\mathrm{p}=0.003$ ) in a subgroup of 91 NAFLD patients.

The Controlled Attenuation Parameter (CAP), implemented on the FibroScan device (Echosens, Paris, France) was firstly used for the noninvasive assessment of steatosis severity and has shown a correlation with histologic grades in adults [2]. In the latest WFUMB guidelines on ultrasound elastography, CAP has been recommended as a point-of-care, standardized and reproducible technique for the detection of liver steatosis [2]. Its accuracy may be affected by variations in cut-off values of different steatosis grades and needs to be better validated in patients with NAFLD with the XL-probe [3]. 
Zeno Sparchez Complex US evaluation of non-alcoholic fatty liver disease. "One stop shop" with new US-based techniques.

In the last few years ultrasound system manufacturers have developed technologies to quantify the ultrasound beam attenuation incorporated into standard ultrasound systems [3-5]. The advantage of such systems is that they can quantify steatosis during a standard B mode liver ultrasound examination when the liver has steatotic features ("bright liver" with posterior attenuation and an increased hepato-renal index).

In this issue of Medical Ultrasonography Bende at al assessed the accuracy of ultrasound-guided attenuation parameter (UGAP) for the quantification of liver steatosis in patients with NAFLD using CAP as the reference method [6]. UGAP measures the attenuation coefficient based on a reference phantom that includes glass bead particles of attenuating materials with known attenuation coefficient [6]. The authors classified the cohort by means of CAP into the following groups: S0 (no steatosis), S1 (mild), S2 (moderate) and S3 (severe). The mean UGAP values increased with the steatosis grade and a very good positive correlation was found between UGAP and CAP values $(\mathrm{r}=0.73, \mathrm{p}<0.0001)$ [6]. This method seems to provide an objective estimation of liver steatosis severity but is also a very good method to assess the efficacy of treatments during follow-up

As many ultrasound systems have incorporated modules for steatosis and also fibrosis quantification, we have reached the time when such "multiparametric" ultrasound systems may enter clinical practice providing important and valuable information regarding the severity of steatosis and fibrosis in the NAFLD population. The examination comes shortly after a normal US examination so in a few minutes not only hepatologists but also internal medicine or ultrasound specialists may have a complex evaluation of a steatotic liver.

\section{References}

1. Zhou JH, Cai JJ, She ZG, Li HL. Noninvasive evaluation of nonalcoholic fatty liver disease: Current evidence and practice. World J Gastroenterol. 2019;25:1307-1326.

2. Sporea I, Bâldea V, Lupușoru R, et al. Quantification of Steatosis and Fibrosis using a new system implemented in an ultrasound machine. Med Ultrason. 2020;22:265271.

3. Castera L, Friedrich-Rust M, Loomba R. Noninvasive assessment of liver disease in patients with nonalcoholic fatty liver disease. Gastroenterology. 2019;156:1264-1281.

4. Ballestri S, Romagnoli D, Nascimbeni F, Francica G, Lonardo A. Role of ultrasound in the diagnosis and treatment of nonalcoholic fatty liver disease and its complications. Expert Rev Gastroenterol Hepatol. 2015;9:603-627.

5. Ozturk A, Grajo JR, Gee MS, et al. Quantitative hepatic fat quantification in non-alcoholic fatty liver disease using ultrasound-based techniques: a review of literature and their diagnostic performance. Ultrasound Med Biol. 2018;44:2461-2475.

6. Bende F, Sporea I, Șirli R, et al. Ultrasound-Guided Attenuation Parameter (UGAP) for the quantification of liver steatosis using the Controlled Attenuation Parameter (CAP) as the reference method. Med Ultrason. 2021;23:7-14. 